referred to our thyroid and endocrine clinics, and indeed our experience would support D Cooke's "suspicion that they represent the tip of an iceberg."

It has been rightly emphasised that primary thyroidal failure is a graded phenomenon, ${ }^{5}$ and in contrast to the frank clinical picture of hypothyroidism in Dr Cooke's patients we have seen several patients who presented with mild and non-specific symptoms following an uneventful pregnancy. Such patients are often reassured their tiredness is due to the "stress and strain" of motherhood or they are prescribed iron treatment despite the absence of any evidence of such deficiency. The subsequent improvement observed tends to perpetuate this practice, although the clinical course merely reflects the transient nature of the thyroid disturbance. The underlying thyroid dysfunction can be easily overlooked in the absence of the characteristic symptom and signs. We have encountered patients subsequently proved to have primary subclinical hypothyroidism in whom the sole presentation was that of persistent inappropriate galactorrhoea 9-12 months after a norma delivery. ${ }^{6}$ In three of our patients the primary subclinical hypothyroidism was transient and two became pregnant within 3-6 months of achieving spontaneously the euthyroid state.

It would be ideal to screen all patients six months after corfinement as advocated by Dr Cooke, but such a scheme is beyond the clinica and laboratory resources of the NHS. We agree with the guidance given in your leading article that a selective group of patients should be carefully watched, and in our experience these include women known to have circulating thyroid antibodies, a family history of autoimmune thyroid disease, or an increase in size of the thyroid gland following pregnancy.

J How

P D BEWSHER

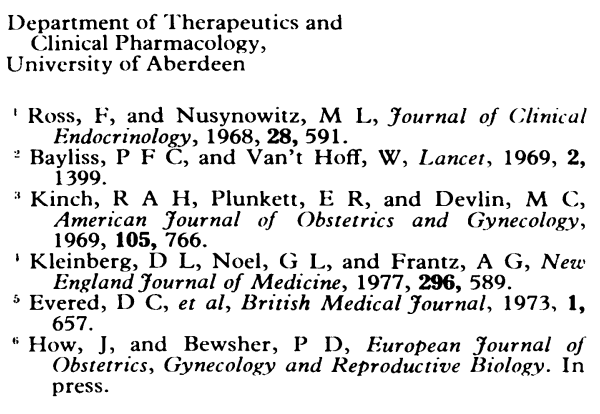

Review of patients after ${ }^{131}$ I treatment for thyrotoxicosis

SIR,-We were interested to see the report from Dr A D Toft and others (21 October, p 1115). Studies which will promote the development of appropriate long-term surveillance programmes for patients treated with radioiodine should be encouraged, but in view of the data which the authors present we feel that their conclusion (namely, that the optimum follow-up interval for postradioiodine patients with normal serum thyrotrophin (TSH) levels is three years) cannot be accepted as a practicable working guideline. Their sample of patients is the end result of a considerable number of selection procedures; in particular the post-treatment interval of 6 to 18 years indicates that these patients are survivors of many events, including hypothyroidism. It is not indicated how the post-treatment intervals are distributed in the geographical subsamples. It is probably not justifiable to extrapolate these results to the management of patients in the first six years after treatment. Losses exceeding $11 \%$ in the four-year study is an important feature worth noting when interpreting the results.

The finding in this study given most prominence is the occurrence of overt hypothyroidism in only one patient, in the group with normal initial serum TSH levels, after four years' follow-up. It is difficult to draw any serious conclusions about one event of this kind occurring in a relatively small sample of 31 patients. However, taking this figure in isolation we might conclude that the expected incidence of overt hypothyroidism at four years is $3.2 \%$, with $95 \%$ confidence limits of $0.08 \%$ to $16.7 \%$. The presence of a raised TSH level in only one of the seven lost patients would, of course, make a big difference to the outcome of the study.

What is probably much more important is the development of a raised TSH level in 19 out of the original 61 patients, in which state the risk of overt hypothyroidism is shown to be high. We have applied a follow-up life-table analysis to their data to show that the five-year cumulative incidence of hypothyroidism in the presence of a raised serum TSH concentration is $20 \cdot 7 \pm 5 \cdot 2(\mathrm{SE}) \%$ and that the cumulative incidence of a raised TSH level in those with an initial normal level is $35.9 \% \pm 6.5$ (full details of the calculations on application). It is not possible to quantify precisely the risk of hypothyroidism for this latter group, because we do not know how long the TSH levels had been raised in those who became hypothyroid in the first group. However, it is clear that an arbitrary three-year follow-up interval may not offer sufficient protection to many patients who have a normal TSH level at a particular point in time. Plans for the long-term surveillance of patients with thyroid disease must be evaluated carefully and shown to be cost-effective, but solutions to the problem are unlikely to be found in small ad hoc studies from which large numbers of patients are lost.

A J Hedley Department of Community Health,

J C G PEarson University of Nottingham

\section{Incidence of congenital rubella}

SIR,-In the parliamentary news section of your issue of 18 November (p 1441) you refer to the reply by the Secretary of State to a question on congenital rubella put by $\mathrm{Mr}$ Lewis Carter-Jones. In your report you include the statement that "the average annual number of children affected by congenital rubella is 400 " and from the text it might be supposed that this was a correct figure accepted by the Secretary of State.

The relevant paragraph of the reply given by the Secretary of State runs as follows

"The report which I received from the Children's Committe in September suggested that the number of infants born this coming winter with congenital rubella could, as a result of the recent steep increase in the incidence of the disease, possibly rise to 1500 to 2000 . This figure was based on the assumption that the average annual number of children affected by congenital rubella was 400 ; while there is no firm evidence on which to base statistics, a surveillance scheme which has been in progress since 1971 suggests that that figure is probably twice as high as the true figure even without allowing for the effect of pregnancy terminations." (Hansard, vol 957, col 110-2)

H YeLLOWLEES Chief Medical Officer Social Security

London SE1

***We regret that this reply was condensed for publication in such a way as to give a wrong impression.-ED, $B M \mathcal{H}$.

\section{Distalgesic and paracetamol poisoning}

SIR,-We cannot accept the views of Drs B A Gennery and R A Lucas (28 October, p 1226) regarding the apparent safety of dextropropoxyphene and their denial of its being the frequent cause of sudden death. Their opinion was based on hospital experience in a small area of Manchester and all overdose cases reported to the coroner in that city over one year (approximate population $\frac{1}{2}$ million). Dextropropoxyphene is a widely used and effective analgesic drug, most commonly prescribed in combination with paracetamol as Distalgesic (paracetamol $325 \mathrm{mg}$ and dextropropoxyphene $32.5 \mathrm{mg}$ ).

In the Midlands (estimated population 5 million) quite a different picture appears. The Home Office Forensic Science Laboratory in Birmingham does many of the analyses in the area in cases of possible overdose. In the last 20 months there were 34 cases in which dextropropoxyphene had been the principal cause of death and only three cases were attributed to paracetamol alone. These were all sudden deaths occurring outside hospital. All these persons except one had taken dextropropoxyphene in the form of Distalgesic. Analysis was carried out by quantitative ultraviolet spectroscopy, quantitative thin-layer chromatography, and latterly by high-performance liquid chromatography on samples from stomach contents, blood, and liver. The three methods corresponded and therefore confirmed their accuracy. About half these persons had also taken alcohol.

The findings from the Midlands agreed with those from Belfast, ${ }^{1}$ where 30 sudden deaths due to dextropropoxyphene abuse occurred in three years. The possibility of Distalgesic causing sudden death by respiratory depression is well known to coroners, forensic scientists, and pathologists, who recognise the characteristic cyanosis and acute pulmonary congestion at necropsy. Death is rapid and usually occurs before medical treatment is available. The Forensic Science Laboratory finds that dextropropoxyphene is the commonest individual drug to cause death. It is not to be confused with paracetamol, which, in contrast, usually causes death from hepatorenal failure after several days. The contradictory reports from Manchester could be explained by different local prescribing habits, but close study of a report ${ }^{2}$ from a Manchester hospital does not confirm this. Dextropropoxyphene deaths usually occur outside hospital and would not be included in any hospital figures. Analysis too can be confusing, since paracetamol, which is easily detected, may mask the presence of dextropropoxyphene, so that unless special methods of separation are employed the presence of significant quantities of dextropropoxyphene may be overlooked.

The tragedy is that persons, particularly 